\title{
DESIGN OF CRITICALLY SUBSAMPLED DFT FILTER-BANKS WITH ALLPASS POLYPHASE FILTERS AND NEAR-PERFECT RECONSTRUCTION
}

\author{
Heinrich W. Löllmann and Peter Vary \\ Institute of Communication Systems and Data Processing (ind) \\ RWTH Aachen University, 52056 Aachen, Germany \\ \{loellmann, vary\}eind.rwth-aachen.de
}

\begin{abstract}
A new design for a recursive DFT analysis-synthesis filter-bank (AS FB) with critical subsampling and near-perfect reconstruction is proposed. The analysis filter-bank consists of allpass polyphase filters to achieve a high frequency selectivity with a low algorithmic complexity and low signal delay. The condition for perfect reconstruction (PR) leads to either unstable or anti-causal synthesis filters. This problem is solved by stable allpass polyphase synthesis filters which are designed by analytical closed-form expressions. The first design can achieve arbitrarily small aliasing, amplitude and phase distortions in dependence of the tolerable signal delay and algorithmic complexity. The second design avoids aliasing and amplitude distortions and minimizes the phase distortions at the expense of an increased system delay. The proposed IIR filter-bank possesses also a significantly lower algorithmic complexity than comparable FIR filter-banks.
\end{abstract}

Index Terms - DFT filter-bank, allpass polyphase filters, IIR phase equalizer, closed-form design, low complexity

\section{INTRODUCTION}

Critically subsampled analysis-synthesis filter-banks (AS FBs) are used for a variety of applications such as compression or subband coding systems, e.g., [1,2]. In most cases, FIR AS FBs are used whose design is well explored, e.g., [3,4]. However, the use of recursive filter-banks is an interesting alternative due to their low algorithmic complexity and signal delay. One design approach for an IIR polyphase network (PPN) DFT filter-bank is to use allpass filters for its polyphase components $[5,6]$. Such a filter-bank can be used for subband coding of images or audio signals $[6,7]$.

Perfect reconstruction (PR) of the input signal can be achieved by a PPN synthesis filter-bank whose polyphase filters consist of the inverse allpass polyphase filters of the analysis filter-bank. This straightforward approach leads to either unstable or non-causal synthesis filters, which can be (approximately) implemented by different techniques. In [6], the finite-length of image signals is exploited to implement the synthesis filters in a non-causal fashion. For the processing of infinite-length signals, such as speech and audio signals, a double buffering scheme can be used [8]. This approach causes a fairly high system delay and requires the transmission of initial filter states to the synthesis filter-bank, which is disadvantageous for audio subband coding, cf., [7]. The transmission of such side information can be avoided by the modified scheme of [7] at the price of a further increased computational complexity and signal delay.

A closed-form design for a causal FIR synthesis filter-bank is presented in [9]. This design achieves near-perfect reconstruction (which is sufficient for many applications) and avoids the problem of non-causal filters.

In this paper, we present an analytic closed-form design for a causal and stable synthesis filter-bank with allpass polyphase filters. In contrast to the approach of [9], aliasing and amplitude distortions can be avoided and a significantly lower algorithmic complexity is achieved. The remaining signal distortions can be made arbitrarily small in dependence of the tolerable signal delay and computational complexity, respectively.

\section{ALLPASS-BASED DFT ANALYSIS FILTER-BANK}

The general structure of a critically subsampled AS FB with $M$ channels is shown in Fig. 1. The analysis subband filters of a DFT filter-bank are complex modulated versions of a prototype lowpass filter according to

$$
H_{i}(z)=H\left(z W_{M}^{i}\right) ; \quad i \in\{0,1 \ldots, M-1\}
$$

with $W_{M}=\exp \{-j 2 \pi / M\}$. Here, a recursive prototype lowpass filter is considered whose PPN representation is given by

$$
H(z)=\frac{1}{M} \sum_{\lambda=0}^{M-1} z^{-\lambda} A_{\lambda}\left(z^{M}\right) .
$$

Its $M$ polyphase components are different allpass filters of order $K_{\lambda}$ with transfer functions

$$
\begin{aligned}
& A_{\lambda}(z)=\prod_{k=1}^{K_{\lambda}} \frac{1-\alpha_{k}(\lambda) z}{z-\alpha_{k}(\lambda)} ;-1<\alpha_{k}(\lambda)<1 \\
& \max _{k, \lambda}\left\{\left|\alpha_{k}(\lambda)\right|\right\}<|z| ; \quad \lambda \in\{0,1, \ldots, M-1\}
\end{aligned}
$$

where $A_{\lambda}(z)=1$ for $K_{\lambda}=0$. The (real) coefficients of the allpass polyphase components for this prototype lowpass filter can

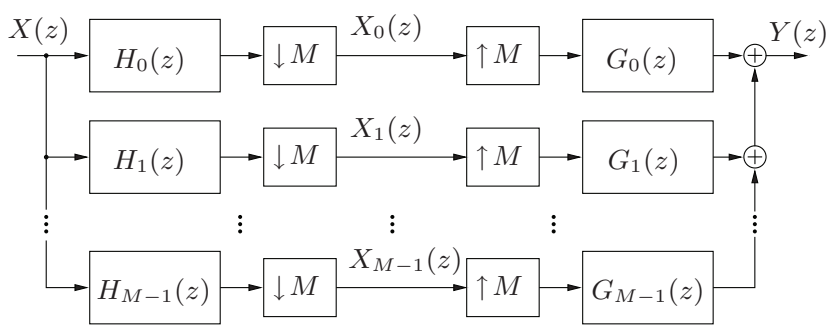

Fig. 1. General structure of a uniform analysis-synthesis filter-bank (AS FB) with $M$ subbands and critical subsampling. 


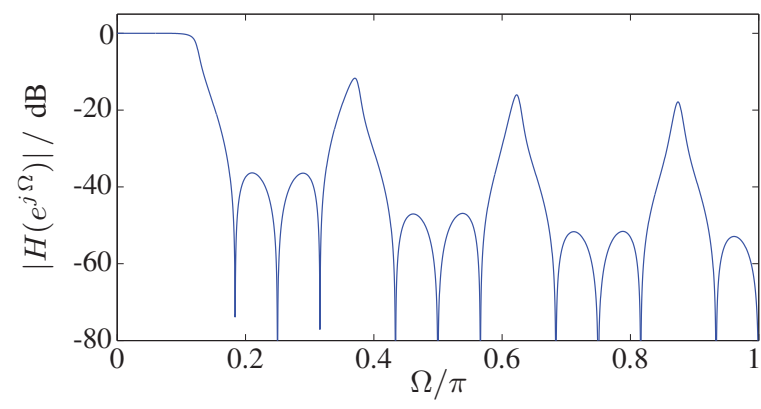

Fig. 2. Magnitude response of the IIR analysis prototype lowpass filter with design parameters $M=8$ and $\Omega_{\mathrm{p}}=0.6 \pi / M$.

be determined by the algorithm presented in [5]. An example for such a prototype filter is provided by Fig. 2, which has been determined by the non-linear phase design of [5] for a passband edge frequency of $\Omega_{\mathrm{p}}=0.6 \pi / M$ and allpass filter degrees $K_{\lambda}=1$ for $\lambda \in\{0,1, \ldots, M-2\}$ and $K_{M-1}=0$. The width of the inevitable side-lobes and the transition-band can be reduced at the price of a diminished stopband attenuation and vice versa. However, the frequency selectivity of such an IIR prototype filter is significantly higher than for a comparable FIR prototype filter.

Eq. (1) and (2) lead to the following PPN representation for the analysis subband filters

$H_{i}(z)=\frac{1}{M} \sum_{\lambda=0}^{M-1} z^{-\lambda} A_{\lambda}\left(z^{M}\right) W_{M}^{-\lambda i} ; i \in\{0,1, \ldots, M-1\}$.

The corresponding PPN implementation of the analysis filter-bank is shown in Fig. 3 (cf., [4]). The inverse discrete Fourier transform (IDFT) can be efficiently calculated by the inverse fast Fourier transform (IFFT), e.g., [10]. The $M$ maximally decimated subband signals can be expressed in the $z$-domain as follows

$$
X_{i}(z)=\frac{1}{M^{2}} \sum_{r=0}^{M-1} X\left(z^{\frac{1}{M}} W_{M}^{r}\right) \sum_{\lambda=0}^{M-1} z^{-\frac{\lambda}{M}} W_{M}^{-\lambda(i+r)} A_{\lambda}(z)
$$

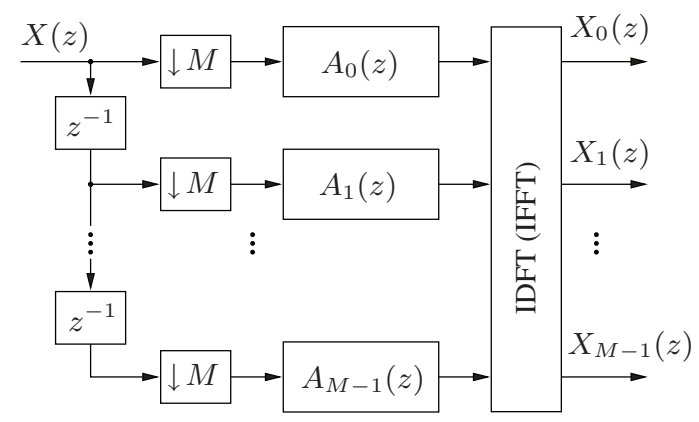

Fig. 3. Polyphase network (PPN) implementation of the critically subsampled DFT analysis filter-bank.

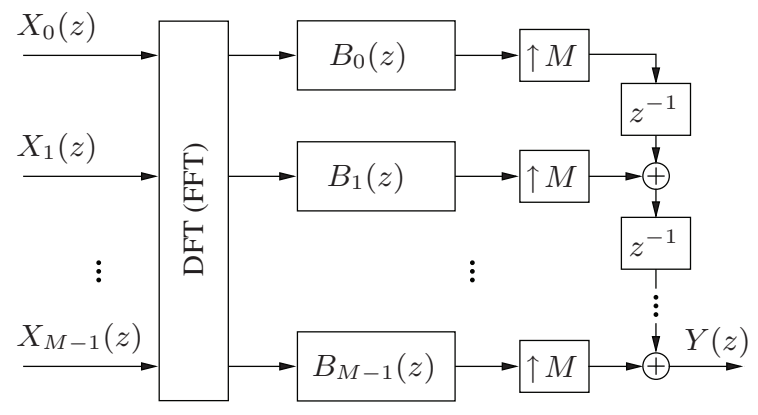

Fig. 4. PPN DFT synthesis filter-bank with upsampling.

\section{SYNTHESIS FILTER-BANK DESIGN}

\subsection{General Concept}

The general structure of the PPN synthesis filter-bank is depicted in Fig. 4. Some straightforward calculations lead to the following $z$-domain representation for the output signal

$$
\begin{aligned}
Y(z) & =\frac{z^{-M+1}}{M} \sum_{r=0}^{M-1} X\left(z W_{M}^{r}\right) \sum_{\lambda=0}^{M-1} A_{\lambda}\left(z^{M}\right) B_{\lambda}\left(z^{M}\right) W_{M}^{-\lambda r} \\
& =z^{-(M-1)}(X(z) \underbrace{\frac{1}{M} \sum_{\lambda=0}^{M-1} A_{\lambda}\left(z^{M}\right) B_{\lambda}\left(z^{M}\right)}_{=T_{\operatorname{lin}}(z)} \\
& +\sum_{r=1}^{M-1} X\left(z W_{M}^{r}\right) \underbrace{\frac{1}{M} \sum_{\lambda=0}^{M-1} A_{\lambda}\left(z^{M}\right) B_{\lambda}\left(z^{M}\right) W_{M}^{-\lambda r}}_{=U_{r}(z)})
\end{aligned}
$$

The function $T_{\text {lin }}(z)$ marks the linear transfer function of the AS FB and $U_{r}(z)$ represents the $r$-th aliasing component due to the subsampling. The peak aliasing distortions of the AS FB are given by [4]

$$
\mathcal{E}_{\mathrm{A}_{\text {peak }}}(\Omega)=\sqrt{\sum_{r=1}^{M-1}\left|U_{r}\left(e^{j \Omega}\right)\right|^{2}} .
$$

Eq. (6) reveals that the condition

$$
A_{\lambda}(z) B_{\lambda}(z) \stackrel{!}{=} z^{-D} \forall \lambda \in\{0,1, \ldots, M-1\}
$$

leads to an AS FB with PR having an overall signal delay of $\tau_{\mathrm{o}}=M-1+M D$ sample instants. This condition can be fulfilled by the choice $B_{\lambda}(z)=A_{\lambda}\left(z^{-1}\right)$ which, however, corresponds to a causal, unstable filter for a region of convergence (ROC) given by $\max \left\{\left|\alpha_{k}(\lambda)\right|\right\}<|z|$, and a stable but anti-causal filter for a ROC $\max \left\{\left|\alpha_{k}(\lambda)\right|\right\}>|z|$. In the following, an alternative design will be devised to avoid the drawbacks associated with non-causal filtering pointed out before.

Instead of a filter inversion problem, Eq. (8) can also be interpreted as a phase equalization problem: The non-linear phase response of the allpass filters $A_{\lambda}(z)$ shall be linearized by a phase equalizer $B_{\lambda}(z)$. One method to obtain such a phase equalizer is to employ a numerical design approach to construct an allpass filter with prescribed phase response, e.g., $[11,12]$. However, such techniques have a relatively high design complexity and do not always 
provide a stable allpass filter. To circumvent these problems, we use the following closed-from design for a stable allpass phase equalizer

$$
P_{\mathrm{AP}}^{(\lambda)}(z)=\prod_{k=1}^{K_{\lambda}} \prod_{n=0}^{N_{\lambda}(k)-1} \frac{1+\alpha_{k}(\lambda)^{2^{n}} z^{2^{n}}}{z^{2^{n}}+\alpha_{k}(\lambda)^{2^{n}}} ; \quad N_{\lambda}(k) \in \mathbb{N} \backslash\{0\}
$$

which has been originally devised in [13] for the construction of allpass transformed DFT filter-banks. ${ }^{1}$ The transfer functions of allpass filter and phase equalizer are given by

$$
\begin{aligned}
T_{\mathrm{AP}}^{(\lambda)}(z) & =A_{\lambda}(z) \cdot P_{\mathrm{AP}}^{(\lambda)}(z) \\
& =\prod_{k=1}^{K_{\lambda}} \frac{1-\alpha_{k}(\lambda)^{d_{\lambda}(k)} \cdot z^{d_{\lambda}(k)}}{z^{d_{\lambda}(k)}-\alpha_{k}(\lambda)^{d_{\lambda}(k)}} ; \quad d_{\lambda}(k)=2^{N_{\lambda}(k)}
\end{aligned}
$$

and represents allpass filters of order

$$
D_{\lambda}=\sum_{k=1}^{K_{\lambda}} d_{\lambda}(k) \forall \lambda \in\{0,1, \ldots, M-1\} .
$$

These allpass filters are always stable since $\left|\alpha_{k}(\lambda)\right|<1$ and tend to a pure delay of $z^{-D_{\lambda}}$ for growing values $d_{\lambda}(k)$. It should be noted that the allpass property is maintained even for quantized allpass coefficients, and that the poles of the allpass phase equalizer of Eq. (9) are closer to the origin than for the allpass filters of Eq. (3).

The proposed phase equalizer of Eq. (9) has a filter degree of $\sum_{k=1}^{K_{\lambda}}\left(d_{\lambda}(k)-1\right)$ and needs $2 \sum_{k=1}^{K_{\lambda}} \log _{2} d_{\lambda}(k)$ real multipliers, $2 \sum_{k=1}^{K_{\lambda}} \log _{2} d_{\lambda}(k)$ real adders and $\sum_{k=1}^{K_{\lambda}}\left(d_{\lambda}(k)-1\right)$ delay elements. ${ }^{2}$ In contrast, a general allpass phase equalizer with the same degree requires $2 \sum_{k=1}^{K_{\lambda}} d_{\lambda}(k)$ multipliers, $2 \sum_{k=1}^{K_{\lambda}} d_{\lambda}(k)$ adders and $\sum_{k=1}^{K_{\lambda}} d_{\lambda}(k)$ delay elements. The corresponding FIR phase equalizer of [9] requires $\sum_{k=1}^{K_{\lambda}} d_{\lambda}(k)$ multipliers, $\sum_{k=1}^{K_{\lambda}}\left(d_{\lambda}(k)-1\right)$ adders and $\sum_{k=1}^{K_{\lambda}}\left(d_{\lambda}(k)-1\right)$ delay elements.

\subsection{Design I}

Near-perfect reconstruction can be accomplished by means of the following allpass polyphase synthesis filters

$$
B_{\lambda}(z)=P_{\mathrm{AP}}^{(\lambda)}(z) \cdot z^{-\left(D_{\max }-D_{\lambda}\right)} \text { with } D_{\max }=\max _{\lambda}\left\{D_{\lambda}\right\} .
$$

Inserting Eq. (12) into Eq. (6) yields the linear transfer function

$$
T_{\text {lin }}(z)=\frac{1}{M} z^{-M D_{\max }} \sum_{\lambda=0}^{M-1} T_{\mathrm{AP}}^{(\lambda)}\left(z^{M}\right) \cdot z^{M D_{\lambda}}
$$

and aliasing components

$$
U_{r}(z)=\frac{1}{M} z^{-M D_{\max }} \sum_{\lambda=0}^{M-1} T_{\mathrm{AP}}^{(\lambda)}\left(z^{M}\right) \cdot z^{M D_{\lambda}} \cdot W_{M}^{-\lambda r}
$$

\footnotetext{
${ }^{1}$ This phase equalizer concept can also be applied in case of complex allpass filter coefficients.

${ }^{2}$ The cascade allpass filter structure is considered consisting of realvalued allpass filters of first order each implemented by two multipliers, two adders and one delay element.
}

with $T_{\mathrm{AP}}^{(\lambda)}(z)$ given by Eq. (10). The linear and aliasing distortions can be made arbitrarily small by using higher values for $d_{\lambda}(k)$. The overall signal delay of the filter-bank amounts to

$$
\tau_{\mathrm{o}}=M-1+M D_{\max }
$$

sample instants.

\subsection{Design II}

Inspection of Eq. (6) reveals that complete aliasing cancellation is achieved, if the product $A_{\lambda}(z) B_{\lambda}(z)$ is identical for all $\lambda \in\{0,1, \ldots, M-1\}$. This can be accomplished by the following polyphase synthesis filters

$$
B_{\lambda}(z)=\prod_{\rho=0}^{\lambda-1} T_{\mathrm{AP}}^{(\rho)}(z) \cdot P_{\mathrm{AP}}^{(\lambda)}(z) \cdot \prod_{i=\lambda+1}^{M-1} T_{\mathrm{AP}}^{(i)}(z) .
$$

The linear transfer function is now given by

$$
T_{\text {lin }}(z)=\prod_{\lambda=0}^{M-1} T_{\mathrm{AP}}^{(\lambda)}\left(z^{M}\right) .
$$

This is an allpass filter so that no amplitude distortions occur. The remaining phase distortions can be made arbitrarily small in dependence of the tolerable signal delay, which amounts to

$$
\tau_{\mathrm{o}}=M-1+M \sum_{\lambda=0}^{M-1} D_{\lambda}
$$

sample instants.

\section{DESIGN EXAMPLES}

The design of a maximal decimated DFT filter-bank with $M=8$ subbands is considered whose analysis prototype filter is shown in Fig. 2. For the synthesis filter-bank design, the proposed allpass polyphase filters have been taken. For comparison, the corresponding FIR synthesis filter-bank design of Galijašević and Kliewer [9] is considered for the same filter degrees and signal delay.

For the synthesis filter-bank design I, the filter degrees $D_{\lambda}$ according to Eq. (11) are equal to $[2,4,4,8,8,16,16,0]$. The zero value indicates an 'identity branch' for $\lambda=M-1$ where $A_{M-1}(z)=1$ and $B_{M-1}(z)=z^{-D_{\max }}$. The linear transfer function and the peak aliasing distortions according to Eq. (6) and (7) are plotted in Fig. 5. ${ }^{3}$ The curves for the new design (marked by the solid blue line) reveal almost no amplitude distortions, but slightly higher group delay distortions in comparison to the corresponding FIR design of [9] (marked by the red dotted curve). The peak aliasing distortions according to Eq. (7) are more alternating than for the FIR synthesis filter-bank design but have a similar mean value.

For the synthesis filter-bank design II, the coefficients $D_{\lambda}$ are equal to $[2,4,4,8,8,16,32,0]$. The resulting transfer function is plotted in Fig. 6 . The new design does not cause amplitude and aliasing distortions, but exhibits slightly higher group delay distortions in comparison to the FIR synthesis filter-bank design. The signal delay is considerably higher than for the design II, but still lower than for a comparable synthesis filter-bank implemented by a non-causal filtering approach, cf., [7]. Moreover, the proposed synthesis filter-bank design exhibits a significantly lower algorithmic complexity than the FIR design of [9] (cf., Section 3.1).

\footnotetext{
${ }^{3}$ In practice, a higher signal reconstruction error is often tolerable so that the synthesis filters can have a lower degree than for these design examples.
} 

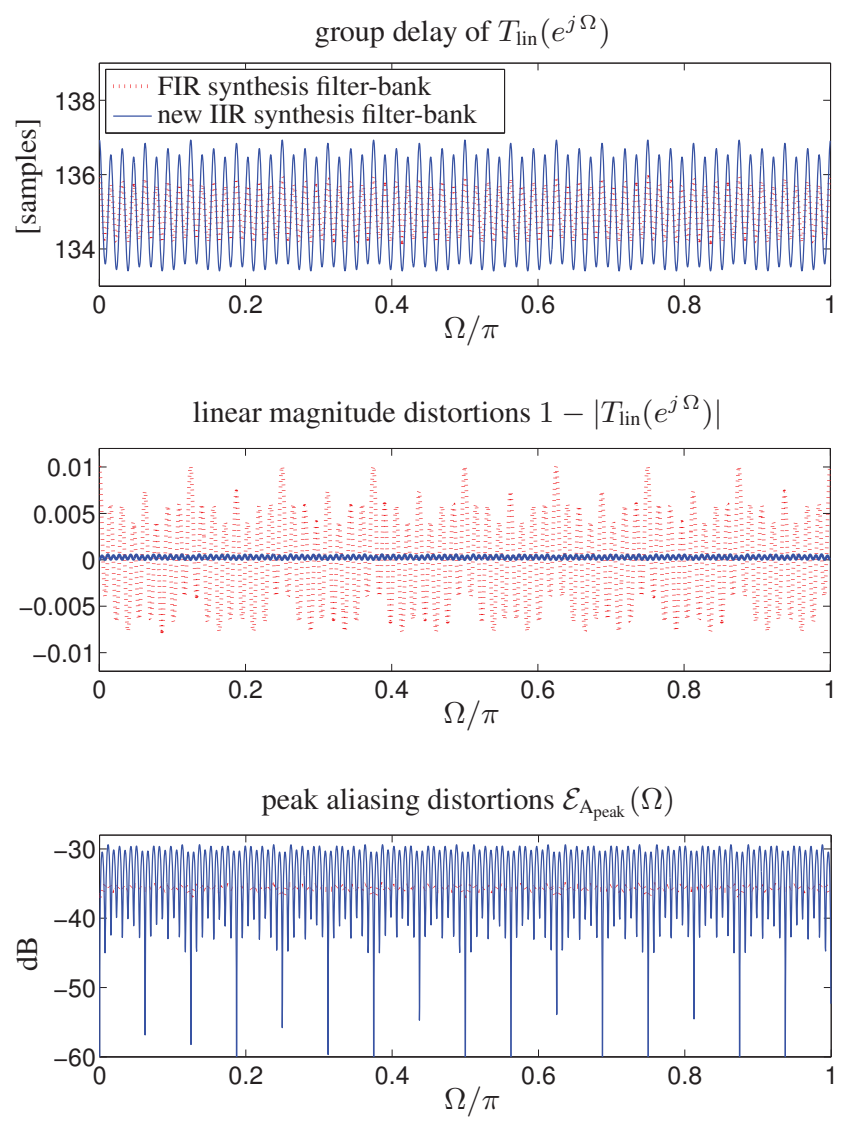

Fig. 5. Analysis of the transfer function of an IIR DFT AS FB with the new IIR synthesis filter-bank design I (blue solid line) and the corresponding FIR synthesis filter-bank of [9] (red dotted line).

\section{CONCLUSIONS}

A novel design for a maximally decimated DFT AS FB with allpass polyphase filters and near-perfect reconstruction is proposed. The synthesis subband filters consist of stable allpass polyphase filters designed by simple closed-form expressions. This allows to control the trade-off between signal distortions and signal delay in a simple and flexible manner. In comparison to the FIR synthesis filter-bank design of [9], a significantly lower algorithmic complexity is accomplished and the filter-bank causes no or negligible amplitude distortions. Aliasing and amplitude distortions can be completely avoided at the expense of a higher signal delay and increased complexity. The slightly higher phase distortions in comparison to an FIR synthesis filter-bank are usually tolerable for speech and audio processing applications due to the insensitivity of the human auditory systems towards moderate phase modifications. The analysis and synthesis subband filters have the same magnitude responses, but the analysis filter-bank has a significantly lower algorithmic complexity than the synthesis filter-bank. Thus, the exchange of analysis and synthesis filter-bank is possible for compression or transmission systems where encoder and decoder have a different computational power. A DFT filter-bank is considered here, but the new approach can be easily applied for other transformation kernels such as the discrete cosine transform (DCT).
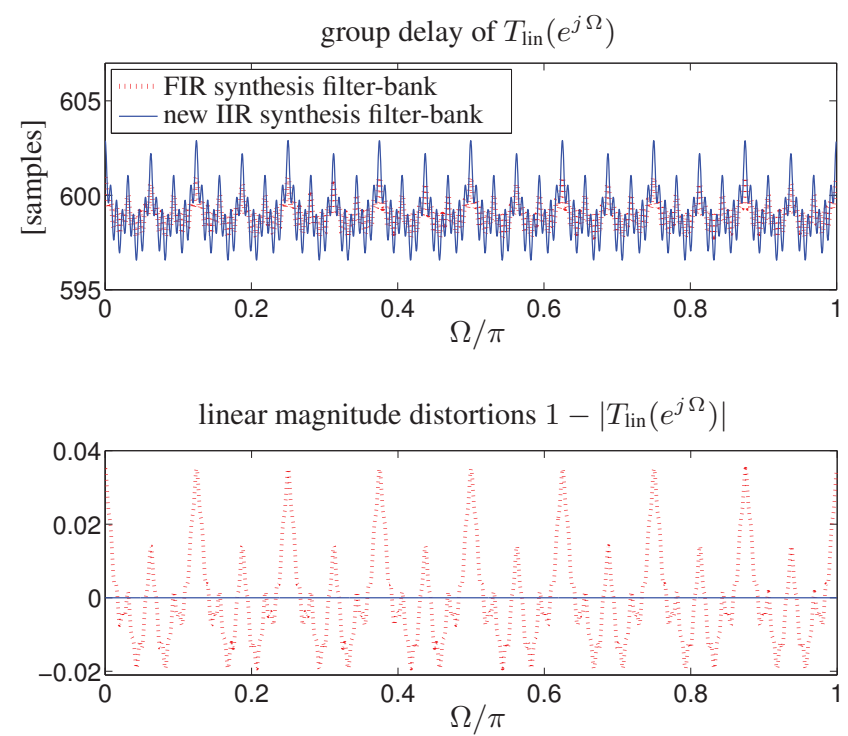

Fig. 6. Analysis of the transfer function of an IIR DFT AS FB with the new IIR synthesis filter-bank design II (blue solid line) and the corresponding FIR synthesis filter-bank of [9] (red dotted line).

\section{REFERENCES}

[1] M. Vetterli and J. Kovačević, Wavelets and Subband Coding, PrenticeHall, Upper Saddle River, New Jersey, 1995.

[2] A. Spanias, T. Painter, and V. Atti, Audio Signal Processing and Coding, Wiley, Hooboken, New Jersey, 2007.

[3] R. E. Crochiere and L. R. Rabiner, Multirate Digital Signal Processing, Prentice-Hall, Englewood Cliffs, New Jersey, 1983.

[4] P. P. Vaidyanathan, Multirate Systems and Filter Banks, Prentice-Hall, Upper Saddle River, New Jersey, 1993.

[5] M. Renfors and T. Saramäki, "Recursive $N$ th-Band Digital FiltersPart I: Design and Properties," IEEE Trans. on Circuits and Systems, vol. 34, no. 1, pp. 24-39, Jan. 1987.

[6] J. H. Husøy and T. A. Ramstad, "Application of an Efficient Parallel IIR Filter Bank to Image Subband Coding," Signal Processing, Elsevier, vol. 20, no. 4, pp. 279-292, Aug. 1990.

[7] C. D. Creusere and S. K. Mitra, "Efficient Audio Coding Using Perfect Reconstruction Noncausal IIR Filter Banks," IEEE Trans. on Speech and Audio Processing, vol. 4, no. 2, pp. 115-123, Mar. 1996.

[8] S. K. Mitra, C. D. Creusere, and H. Babic, "A Novel Implementation of Perfect Reconstruction QMF Banks Using IIR Filters for Infinite Length Signals," in Proc. of Intl. Symposium on Circuits and Systems (ISCAS), San Diego, USA, May 1992, pp. 2312-2315.

[9] E. Galijašević and J. Kliewer, "Design of Allpass-Based NonUniform Oversampled DFT Filter Banks," in Proc. of Intl. Conference on Acoustics, Speech, and Signal Processing (ICASSP), Orlando (Florida), USA, May 2002, vol. 2, pp. 1181-1184.

[10] A. V. Oppenheim, R. W. Schafer, and J. R. Buck, Discrete-Time Signal Processing, Prentice-Hall, Upper Saddle River, New Jersey, 2nd edition, 1999.

[11] M. Lang, "Allpass Filter Design and Applications," IEEE Trans. on Signal Processing, vol. 46, no. 9, pp. 2505-2513, Sept. 1998.

[12] M. Lang and T. I. Laakso, "Simple and Robust Method for the Design of Allpass Filters Using Least-Squares Phase Error Criterion," IEEE Trans. on Circuits and Systems II, vol. 41, no. 1, pp. 40-48, Jan. 1994.

[13] H. W. Löllmann and P. Vary, "Parametric Phase Equalizers for Warped Filter-Banks," in Proc. of European Signal Processing Conference (EUSIPCO), Florence, Italy, Sept. 2006. 